values

$$
\frac{1}{n} \sum_{1}^{n} h_{k}(P) \quad(n=1,2, \cdots)
$$

are majorized by a single integrable function $H(P)$. Therefore, to formula (24) we apply, not Lebesgue's theorem, but Fatou's theorem. Using the inequality (22), we find

$$
\begin{aligned}
\int|\psi(P)| & \leq \lim _{n \rightarrow \infty} \int\left|\frac{1}{n} \sum_{1}^{n} h_{k}(P)\right| \\
& \leq \lim _{n \rightarrow \infty} \frac{1}{n} \sum \int\left|h_{k}(P)\right|=\int\left|h_{1}(P)\right|<\epsilon .
\end{aligned}
$$

Combining our results, we find

$$
\begin{aligned}
\left|\int f_{1}(P)-\int \varphi(P)\right| & =\left|\int g_{1}(P)-\int \chi(P)+\int h_{1}(P)-\int \psi(P)\right| \\
& \leq\left|\int g_{1}(P)-\int \chi(P)\right|+\int\left|h_{1}(P)\right|+\int|\psi(P)| \\
& <0+\epsilon+\epsilon .
\end{aligned}
$$

Letting $\epsilon \rightarrow 0$, we obtain the required result $\int f_{1}(P)=\int \varphi(P)$.

\title{
REFERENCES
}

1. J. Moshman, The generation of pseudo-random numbers on a decimal calculator, J. Assoc. for Computing Machinery 1, No. 2, 88 (1954)

2. J. Todd, Some experiments on the Monte Carlo method, presented before the Institute of Mathematical Statistics, Boston, 1951

3. M. Juncosa, Random number generation on the BRL high-speed computing machines, Rep. No. 855, Ballistic Research Laboratories, Aberdeen Proving Ground, 1953

4. E. Leshan, A general purpose Monte Carlo program, American-Standard Atomic Energy Division Report, 1956

5. H. Weyl, Über die Gleichverteilung von Zahlen mod. Eins., Math. Ann. 77, 313 (1916)

6. F. Riesz, Sur la théorie ergodique, Commentarii Mathematici Helvetici 17, 221 (1945)

7. G. Birkhoff, Proof of the ergodic theorem, Proc. Natl. Acad. Sci. 18, 650 (1932)

8. E. Titchmarsh, The theory of functions, Oxford University Press, 2nd Ed., 1939

\section{SIMULTANEOUS INVARIANCE OF GENERALIZED SPHERICAL HARMONICS UNDER THE OPERATIONS OF TWO ROTATION GROUPS*}

\section{By R. N. D'HEEDENE (Cornell University)}

Abstract. A method is found for evaluating the coefficients of a sum of generalized spherical harmonics so that the sum will be simultaneously invariant under two rotation groups. The coefficients for a sum of ordinary spherical harmonics invariant under each individual group must first be known.

*Received May 15, 1957. 
If a rigid molecule is situated in a lattice, free to rotate but not displace, the molecule's potential energy $V$, a function of its rotational position, must have certain symmetries, dictated by the symmetry of the molecule and the symmetry of the lattice. In this paper a consequence of these symmetry properties of $V$ is discussed.

Let the position of the molecule be described by the three Eulerian angles $(\theta, \phi, \psi)$, of which $\theta$ and $\phi$ are the polar coordinates of a $z$-axis fixed in the molecule with respect to an $(X, Y, Z)$ system of coordinates fixed in the lattice, and $\psi$ describes the rotation of the molecule about $z^{1}$. Let there be a group $G$ of rotations of $(X, Y, Z)$ describing the symmetries of the lattice, and a group $H$ of rotations of $(x, y, z)$ (the molecule-fixed coordinates) describing the symmetry of the molecule. Suppose $V(\theta, \phi, \psi)$, the potential energy, is expressed as a series of generalized spherical harmonics (often called the symmetric top eigenfunctions),

$$
V(\theta, \phi, \psi)=\sum_{J=0}^{\infty} \sum_{K=-J}^{J} \sum_{M=-J}^{J} C_{J}^{K, M} Y_{J}^{K, M}(\theta, \phi, \psi) .
$$

Then the requirement that $V$ be invariant under each operation in $G$ or $H$ will place certain restrictions on the constants $C_{J}^{K, M}$ (the higher the symmetry expressed by $G$ and $H$, the more of them will be zero). We wish to determine these restrictions.

Instead of using the real triplet $(\theta, \phi, \psi)$ to represent Eulerian angles, we will use the complex doublet (or spinor) $(\xi, \eta)$ where

$$
\begin{aligned}
& \xi=\exp [-i(\phi+\psi) / 2] \cos \frac{\theta}{2}, \\
& \eta=\exp [i(\phi-\psi) / 2] \sin \frac{\theta}{2},
\end{aligned}
$$

or even more simply, the matrix, $\Gamma$, where

$$
\Gamma=\left(\begin{array}{rr}
\xi^{*} & \eta^{*} \\
-\eta & \xi
\end{array}\right)
$$

In this notation, if $(x, y, z)$ is given by $\Gamma_{1}$ and $\left(x^{\prime}, y^{\prime}, z^{\prime}\right)$ by $\Gamma_{2}$, each with respect to $(X, Y, Z)$, and $\left(x^{\prime}, y^{\prime}, z^{\prime}\right)$ by $\Gamma$ with respect to $(x, y, z)$, then the relation between the angles is

$$
\Gamma_{2}=\Gamma_{1} \Gamma
$$

an easy result, but one involving some arithmetic ${ }^{2}$.

We will write the generalized spherical harmonics as $Y_{J}^{K, M}(\Gamma)$ where $\Gamma$ indicates the argument $(\theta, \phi, \psi)$ of $Y$.

Note that we may consider (4) as giving the transformation under $\Gamma$ of the Eulerian angles $\Gamma_{1}$ into $\Gamma_{2}$. That is, $\Gamma$ represents a rotation $R(\Gamma)$ in $H$, under which $\Gamma_{1} \rightarrow \Gamma_{2}$ gives the transformation of the Eulerian angles describing $(x, y, z)$ with respect to $(X, Y, Z)$. An important result is that under such a transformation,

$$
R(\Gamma) Y_{J}^{K, M}\left(\Gamma_{1}\right)=Y_{J}^{K, M}\left(\Gamma_{2}\right)=\sum_{S=-J}^{J} Y_{J}^{K, S}\left(\Gamma_{1}\right) Y_{J}^{S, M}(\Gamma)
$$

${ }^{1}$ See Goldstein, H., Classical Mechanics, Addison-Wesley, 1953, p. 107 for an excellent figure showing the Eulerian angles.

2Takahashi, T., J. Phys. Soc. Japan, 7, 307 (1952). 
gives the transformation of the generalized spherical harmonics ${ }^{2}$. Letting $Y_{J}(\Gamma)$ be a square matrix with elements $Y_{J}^{K}, M(\Gamma),(5)$ may be written as

$$
R(\Gamma) Y_{J}\left(\Gamma_{1}\right)=Y_{J}\left(\Gamma_{2}\right)=Y_{J}\left(\Gamma_{1}\right) Y_{J}(\Gamma) \text {. }
$$

The transformation matrix elements for ordinary spherical harmonics are the same as those for generalized spherical harmonics; using essentially the same notation,

$$
R(\Gamma) Y_{J}^{K}\left(\theta_{1}, \phi_{1}\right)=Y_{J}^{K}\left(\theta_{2}, \phi_{2}\right)=\sum_{S} Y_{J}^{S}\left(\theta_{1}, \phi_{1}\right) Y_{J}^{S, K}(\Gamma)
$$

is a well known result ${ }^{3}$.

The above equations express transformations under a rotation $R(\Gamma)$ in $H$. For a transformation $R\left(\Gamma^{\prime}\right)$ in $G$, the situation is slightly different. If we let $R\left(\Gamma^{\prime}\right)(X, Y, Z)=$ $\left(X^{\prime}, Y^{\prime}, Z^{\prime}\right)$, and $\Gamma_{1}, \Gamma_{2}$ locate $(x, y, z)$ with respect to $(X, Y, Z),\left(X^{\prime}, Y^{\prime}, Z^{\prime}\right)$, respectively, then as we see by applying (4) and considering $\left(X^{\prime}, Y^{\prime}, Z^{\prime}\right)$ as the $(X, Y, Z)$ of equation (4), we have

$$
\Gamma_{2}=\left(\Gamma^{\prime}\right)^{-1} \Gamma_{1}
$$

(exponential-1 indicates inversion) replacing (4), so that

$$
R\left(\Gamma^{\prime}\right) Y_{J}\left(\Gamma_{1}\right)=Y_{J}\left(\Gamma_{2}\right)=Y_{J}\left(\left(\Gamma^{\prime}\right)^{-1}\right) Y_{J}(\Gamma)
$$

gives the transformation of the generalized spherical harmonics for elements $R\left(\Gamma^{\prime}\right)$ of $G$.

Now let the sum in (1) be invariant under each $R\left(\Gamma_{i}\right)$ in $H$ and each $R\left(\Gamma_{i}^{\prime}\right)$ in $G$. Then, by (6) and (9), we require for each $R\left(\Gamma_{i}\right)$,

$$
\begin{aligned}
\sum_{J, K, M} C_{J}^{K, M} Y_{J}^{K, M}(\Gamma) & =R\left(\Gamma_{i}\right) \sum_{J, K, M} C_{J}^{K, M} Y_{J}^{K, M}(\Gamma) \\
& =\sum_{J, K, M, S} C_{J}^{K, M} Y_{J}^{S, M}\left(\Gamma_{i}\right) Y_{J}^{K, s}(\Gamma)
\end{aligned}
$$

and, using the fact that if $R\left[\left(\Gamma^{\prime}\right)^{-1}\right]$ is in $G$, then, since $G$ is a group, $R\left(\Gamma^{\prime}\right)$ is in $G$, for each $R\left(\Gamma_{i}^{\prime}\right)$,

$$
\begin{aligned}
\sum_{J, K, M} C_{J}^{K, M} Y_{J}^{K, M}(\Gamma) & =R\left(\Gamma_{i}^{\prime}\right) \sum_{J, K, M} C_{J}^{K, M} Y_{J}^{K, M}(\Gamma) \\
& =\sum_{J, K, M, S} C_{J}^{K, M} Y_{J}^{S, M}(\Gamma) Y_{J}^{K, S}\left(\Gamma_{i}^{\prime}\right) .
\end{aligned}
$$

Equating the coefficients of $Y_{J}^{K, M}(\Gamma)$ in (10) and (11) gives

$$
C_{J}^{K, M}=\sum_{S} C_{J}^{K, s} Y_{J}^{M, s}\left(\Gamma_{i}\right)=\sum_{S} C_{J}^{S, M} Y_{J}^{S, K}\left(\Gamma_{i}^{\prime}\right)
$$

for each $\Gamma_{i}$ in $H, \Gamma_{i}^{\prime}$ in $G$.

Let constant vectors $h_{J}, g_{J}$ be known, such that

$$
Y_{J}\left(\Gamma_{i}\right) h_{J}=h_{J}, \quad Y_{J}\left(\Gamma_{i}^{\prime}\right) g_{J}=g_{J}
$$

for each $\Gamma_{i}, \Gamma_{i}^{\prime}$. It is reasonable to assume such vectors known, since, using group theory to determine the number of identical $\left(A_{1}\right)$ irreducible representation occurring

'Wigner, E. P., Gruppentheorie und ihre Anwendung auf die Quantenmechanik der Atomspektren, Edwards Brothers, 1944. 
in the $2 J+1$ dimensional representation of the group, one can, at least in principle, find coefficients $h_{J}^{R}$ so that the sum

$$
\sum_{K=-J}^{J} h_{J}^{K} Y_{J}^{K}(\theta, \phi)
$$

is invariant under a group $H$ (the same comments obviously hold for $g, G)^{4}$. And, by (7), this gives, for each $\Gamma_{i}$ in $H$,

$$
\begin{aligned}
\sum_{K=-J}^{J} h_{J}^{K} Y_{J}^{K}(\theta, \phi) & =R\left(\Gamma_{i}\right) \sum_{K} h_{J}^{K} Y_{J}^{K}(\theta, \psi) \\
& =\sum_{K, S} h_{J}^{K} Y_{J}^{S}(\theta, \phi) Y_{J}^{S, K}\left(\Gamma_{i}\right) .
\end{aligned}
$$

Equating the coefficients of $Y_{J}^{K}(\theta, \phi)$ in (15) gives (13).

We now hare the solution to our problem; setting

$$
C_{J}^{K, M}=h_{J}^{M} g_{J}^{K *}
$$

makes (1) invariant under both $G$ and $H$, as we see by showing directly that (16) satisfies (12), using (13) and the fact that $Y_{J}$ is Hermitean:

$$
\begin{aligned}
\sum_{S} C_{J}^{K, s} Y_{J}^{M, S}\left(\Gamma_{i}\right) & =\sum_{S} h_{J}^{S} g_{J}^{K *} Y_{J}^{M, S}\left(\Gamma_{i}\right) \\
& =h_{J}^{M} g_{J}^{K *}=C_{J}^{K, M}, \\
\sum_{S} C_{J}^{S, M} Y_{J}^{S, K}\left(\Gamma_{i}^{\prime}\right) & =\sum_{S} h_{J}^{M} g_{J}^{S *} Y_{J}^{S, K}\left(\Gamma_{i}\right) \\
& =g_{J}^{K}{ }^{M} h_{J}^{M}=C_{J}^{K, M} .
\end{aligned}
$$

Generalization of this theorem to finding appropriate $C_{J}^{K, M}$ so that the sum (1), rather than being invariant, will have specified transformation properties, i.e. belong to a representation other than $A_{1}$, is obvious.

As an example the first non-zero $C_{J}^{K, M}$ will be found for the case of a tetrahedron in a cubic lattice (e.g. solid methane). For the cubic group, $A_{1}$ occurs once in the one $(J=0)$ dimensional representation, not at all in the representations corresponding to $J=1,2,3$, and once in the nine $(J=4)$ dimensional representation. Similarly, for the tetrahedral group, $A_{1}$ occurs once in the representations corresponding to each of $J=0$, 3 , 4. It is trivial that $C_{0} \neq 0$. Since no $A_{1}$ representation occurs for $J=1,2$ in either group, $C_{1}=C_{2}=0$. Since $g_{3}=0$ (corresponding to the cubic group), $C_{3}=0$ although $h_{3} \neq 0$. Now the sum

$$
\left(\frac{7}{12}\right)^{1 / 2} P_{4}^{0}(\cos \theta)+\left(\frac{5}{12}\right)^{1 / 2}(2)^{1 / 2} P_{4}^{4}(\cos \theta) \cos 4 \phi
$$

of fourth order ordinary spherical harmonics is invariant under the cubic group ${ }^{4}$ and therefore under the lower-symmetry tetrahedral group. Thus

$$
g_{4}=h_{4}=\left[\left(\frac{5}{24}\right)^{1 / 2}, 0,0,0,\left(\frac{7}{12}\right)^{1 / 2}, 0,0,0,\left(\frac{5}{24}\right)^{1 / 2}\right]^{T}
$$

'Bethe, H. A., Ann. der Physik, ser. 5, 3, 133 (1929). 
and (16) gives

$$
\begin{aligned}
\frac{5}{24}\left(Y_{4}^{4,4}+Y_{4}^{4,-4}+Y_{4}^{-4,4}\right. & \left.+Y_{4}^{-4,-4}\right) \\
& +\left(\frac{35}{288}\right)^{1 / 2}\left(Y_{4}^{4,0}+Y_{4}^{-4,0}+Y_{4}^{0.4}+Y_{4}^{0,-4}\right)+\frac{7}{12} Y_{4}^{0,0}
\end{aligned}
$$

as a properly invariant function.

The author wishes to extend his appreciation to Professor R. Bersohn, without whose assistance this work would have been impossible.

\section{ON THE MOTION OF A SIMPLE PENDULUM*}

\section{BY BORIS GARFINKEL (Ballistic Research Laboratories, Aberdeen Proving Ground, Md.)}

Abstract. The vanishing of the tension in a simple pendulum supported by a flexible cord causes the particle to pass from the circular to a parabolic trajectory. The number and the nature of such transitions are related here to the value of the initial energy.

1. When the initial energy of a simple pendulum lies in a certain interval, the tension vanishes at some instant of the motion. Then, if the support is provided by a flexible cord, the particle passes from the circular to a parabolic trajectory. The number and the nature of such transitions are shown here to be precisely related to a dimensionless energy parameter, $\xi$. Despite the intrinsic interest and the relative simplicity of this motion, it does not appear to have been treated in the literature.

Let $l$ be the length, $m$ the mass of the pendulum, $r$ its distance from the point of support, $\theta$ the angular coordinate measured from the downward-drawn vertical line, and $g$ the acceleration of gravity. The constraint

$$
l-r \geq 0
$$

can be replaced by the condition

$$
\lambda(l-r)=0,
$$

where $\lambda$ is a multiplier vanishing if $l>r$ and admitting a non-zero value if $l=r$. The Lagrangian of the system,

$$
L=\frac{1}{2} m\left(r^{\cdot 2}+r^{2} \theta^{\cdot 2}\right)+m g r \cos \theta+\lambda(l-r),
$$

leads to the differential equations of motion,

$$
m\left(r^{\cdot}-r \theta^{\cdot 2}-g \cos \theta\right)+\lambda=0, \quad r \theta^{\cdot}+2 r^{\cdot} \theta^{\cdot}+g \sin \theta=0,
$$

which together with (2) and (1) determine the functions $r(t), \theta(t), \lambda(t)$ when the initial conditions are prescribed. From (4.1) the multiplier $\lambda(t)$ can be identified with the

*Received July 22, 1957. 